\title{
A cost-effective method for reducing soil disturbance- induced errors in static chamber measurement of wetland methane emissions
}

\author{
R. Scott Winton · Curtis J. Richardson
}

Received: 4 March 2015/ Accepted: 24 October 2015/Published online: 19 November 2015

(C) Springer Science+Business Media Dordrecht 2015

\begin{abstract}
Static chambers used for sampling methane $\left(\mathrm{CH}_{4}\right)$ in wetlands are highly sensitive to soil disturbance. Temporary compression around chambers during sampling can inflate the initial chamber $\mathrm{CH}_{4}$ headspace concentration and/or lead to generation of non-linear, unreliable flux estimates that must be discarded. In this study, we tested an oftenused rubber gasket (RG)-sealed static chamber against a water-filled gutter (WFG) seal design that could be set up and sampled from a distance of $2 \mathrm{~m}$ with a newly designed remote rod sampling system to reduce soil disturbance. Compared to conventional RG design, our remotely sampled static chambers reduced the chance of detecting inflated initial $\mathrm{CH}_{4}$ concentrations $(>3.6 \mathrm{ppm})$ from 66 to $6 \%$ and nearly doubled the proportion of robust linear regressions $\left(r^{2}>0.9\right)$ from 45 to $86 \%$. Importantly, the remote rod sampling system allows for more accurate and reliable $\mathrm{CH}_{4}$ sampling without costly boardwalk construction. This paper presents results demonstrating that the remote rod sampling system combined with WFG static chambers improves $\mathrm{CH}_{4}$ data reliability by reducing initial gas measurement variability due to chamber disturbance when tested on a mineral
\end{abstract}

R. S. Winton $(\bowtie) \cdot$ C. J. Richardson

Nicholas School of the Environment, Duke University

Wetland Center, Duke University, Durham, NC 27708,

USA

e-mail: scott.winton@duke.edu soil-restored wetland in Charles City County, Virginia, USA.

Keywords Gas flux - Greenhouse gas · Methane . Static chamber · Wetland

\section{Introduction}

Methane $\left(\mathrm{CH}_{4}\right)$ is the second most important longlived greenhouse gas (GHG) after carbon dioxide (Myhre et al 2013), with wetlands representing the single most important source to the atmosphere (Bridgham et al 2006). As a result, the estimation of $\mathrm{CH}_{4}$ flux has become an important component of many studies of wetland carbon biogeochemical cycling, with the non-flow-through-non-steady-state chamber method (Livingston and Hutchinson 1995) or "static chamber" method, most popular because of its simplicity and cost-effectiveness.

It is popular to use simple linear regression of chamber headspace concentrations as a function of time to estimate gas emissions' rate (Weishampel and Kolka 2008), but the act of repeated sampling from static chambers can cause soil disturbance and significant deviations from linearity (Forbrich et al 2010). Dissolved $\mathrm{CH}_{4}$ within saturated or inundated wetland soil pores is particularly sensitive to pressure changes, which can lead to $\mathrm{CH}_{4}$ ebullition (Strack et al 2005). Ebullition can occur in the absence of artificial 
disturbance, but disturbance-induced ebullition is typically the cause of non-linear (based on an $r^{2}$ threshold of 0.9) or unusable $\mathrm{CH}_{4}$ flux data. Studies do not always report how much data are discarded due to soil disturbance, but anywhere from $45 \%$ (Nahlik and Mitsch 2010) to $65 \%$ (Morse et al 2012) of static chamber incubations can be affected. Loss of such a high proportion of data is not only resource inefficient but also poses the threat of introducing sampling errors and bias.

After observing persistent evidence that disturbance was affecting our $\mathrm{CH}_{4}$ flux results using a rubber gasket (RG) static chamber (Weishampel and Kolka 2008), we redesigned our static chambers to incorporate a water-filled gutter (WFG) seal (Livingston and Hutchinson 1995; Wang et al 2006; Krauss and Whitbeck 2011), which allows setup and sampling to be conducted from a distance of $2 \mathrm{~m}$ using a remote rod sampling system (RRSS) so as to minimize wetland soil disturbance (Fig. 1). Here we compare reliability data from our WFG static chamber with RRSS compared against those of a more conventional RG static chamber. We also present reliability data

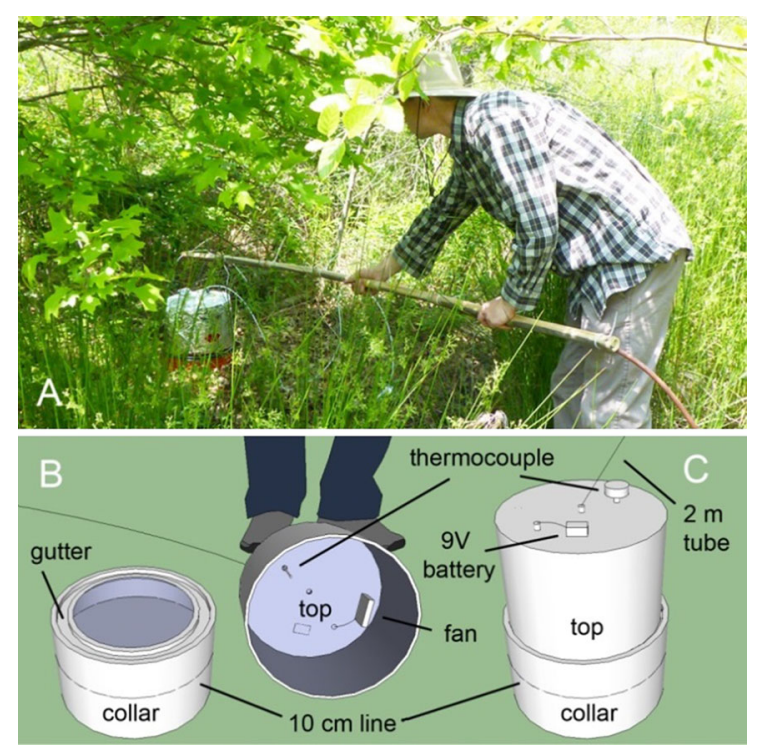

Fig. 1 Illustrations of the water-filled gutter static chamber design that allows the use of remote rod sampling system (RRSS): Photograph of chamber being deployed using RRSS in the Charles City Wetland in Charles City County, Virginia (a); schematic of chamber disassembled to reveal water-fillable gutter on rim of collar that creates an air tight seal, internal fan to mix headspace air and thermocouple to monitor internal chamber temperature $(b)$; schematic of chamber assembled (c) testing whether the WFG seal alone or remote setup using the RRSS in conjunction with the WFG is necessary to improve reliability.

Our findings may help improve field effort efficiency and eliminate boardwalk construction costs for those planning to utilize static chambers for estimation of $\mathrm{CH}_{4}$ gas flux from wetlands.

\section{Methods}

The study took place within the 20.8-hectare Charles City Wetland Mitigation Site, which is located in Charles City County, Virginia, USA, and owned by the Virginia Department of Transportation as part of its compensatory mitigation program (Bailey et al. 2007). Precipitation is the dominant hydrologic input and the CCW may hold up to $0.5 \mathrm{~m}$ of standing water during cooler months (Bailey et al 2007). The soil is mapped as a complex of Chickahominy (fine, mixed, semiactive, thermic Typic Endoaquults) and Newflat (fine, mixed, subactive, thermic Aeric Endoaquults) (Bergschneider 2005). Site history is complex, with one unsuccessful restoration attempt for this formerly forested wetland being followed by an experimental addition of organic matter amendments to improve tree growth. For more detail on site characteristics, see Bruland and Richardson (2004), Bergschneider (2005), and Bailey et al. (2007).

In late summer 2011, we imbedded a $20-\mathrm{cm}$ diameter PVC collar 10-15 cm into the soil in each of 20 plots ( 4.6 by $3.1 \mathrm{~m}$ ) for RG static chamber trace gas sampling. We set up the RG static chambers by placing a PVC cap with a rubber gasket over beveled collar tops by hand. Mean assembled RG chamber height was approximately $13.5 \mathrm{~cm}$ corresponding to a volume of $>4 \mathrm{~L}$. We sampled gas using RG static chambers from 20 collars in September and October 2011 and from 27 collars in February 2012 for a total of 67 sets of gas measurements.

In spring of 2012, we imbedded 45-cm-diameter plastic collars affixed with water-fillable gutters to a depth of $10-15 \mathrm{~cm}$ in the same 20 plots for WFG static chamber trace gas sampling using RRSS. Both WFG and RG chamber caps are equipped with an internal computer fan powered by a 9 -volt battery to circulate chamber headspace as recommended by Christiansen et al (2011) and a thermocouple allowing for internal chamber temperature $(\mathrm{T})$ to be recorded during each 
sample extraction. We coated the WFG caps with reflective aluminum foil to minimize solar warming as recommended by the US Department of Agriculture (Parkin and Venterea 2010).

We set up and sampled WFG static chambers from a distance of approximately $2 \mathrm{~m}$ using our RRSS in which we fill the gutter with water using a 2-m polyethylene tube and place the plastic top using a 2-m rod. We then extracted air samples using a plastic syringe via the 2-m, 1-mm-inner diameter plastic tube following flushing to mix tube air with chamber headspace (total tube volume is $1.6 \mathrm{~mL}$-approximately $0.01 \%$ of headspace volume). The mean height of assembled WFG chamber was $23 \mathrm{~cm}$ with headspace volume ranging from approximately $10 \mathrm{~L}$ during periods of high water to $16 \mathrm{~L}$ during dry periods. We sampled $\mathrm{CH}_{4}$ gas using WFG static chambers from 20 collars in May, July, September (twice), and November 2012 and February 2013 for a total of 120 sets of gas measurements. During one of the two sampling dates in September 2012, we sampled 10 of the 20 WFG static chambers without using the RRSS for setup. Instead we simply walked up to fill the gutter and placed the chamber top by hand (the traditional method, required when using RG) to test for disturbance during WFG setup without RRSS.

After setup of both RG and WFG static chambers, we immediately extracted a 50-mL headspace sample via a plastic syringe and deposited it into a mylar gastight sample bag. We recorded ambient air temperature (T), internal chamber $\mathrm{T}$, soil $\mathrm{T}$ at $5 \mathrm{~cm}$ depth for initial and subsequent samples taken approximately 5 , 15 , and $30 \mathrm{~min}$ following chamber setup. Total extracted sample volume $(200 \mathrm{~mL})$ was never more than $5 \%$ of total headspace volume and acts to counterbalance the pressure buildup from emitted gases over $30 \mathrm{~min}$ of incubation. We transported gas bags to the Duke University Wetland Center laboratory and analyzed within one week of sampling on a Varian 450 Gas Chromatograph (GC) equipped with a flame ionization detector. We analyzed duplicates of all samples, and when duplicate values differed by $<10 \%$, we used the mean for flux calculations.

In addition to $\mathrm{CH}_{4}$, we simultaneously measured carbon dioxide $\left(\mathrm{CO}_{2}\right)$ concentrations using a methanizer in our $\mathrm{GC}$, allowing us to test for static chamber sampling effects on $\mathrm{CO}_{2}$. Since $\mathrm{CO}_{2}$ is approximately 50 times more soluble in water, it is far less susceptible to disturbance-induced ebullition effects and did not manifest obvious performance differences between RG and WFG chambers with or without the RRSS system. Therefore, we limit the scope of this paper to the effects we observed on $\mathrm{CH}_{4}$ reliability. $\mathrm{CH}_{4}$ flux estimates at the CCW (described in detail in Winton and Richardson 2015) range from below detection limits to as high as approximately $5 \mathrm{mg} \mathrm{m}^{-2} \mathrm{~h}^{-1}$.

We consider two performance metrics in our evaluation of chamber reliability: (1) the initial headspace $\mathrm{CH}_{4}$ concentration $\left(C_{\text {initial }}\right)$ as sampled immediately after chamber setup, with higher values indicative of more soil disturbance during chamber setup, and (2) $r^{2}$ of the calculated regression line.

We separately analyzed data from one of our two sampling dates in September 2012 when 10-20 plots were sampled without using the RRSS for WFG static chamber setup, to evaluate the importance of the RRSS in improving data reliability over WFG static chambers setup by hand.

To test for differences in mean $C_{\text {initial }}$ between RG $(n=67)$ and WFG with RRSS $(n=100)$ static chamber methods, and between WFG with and without the RRSS, we use Welch's $t$ test on data log transformed to better meet the assumption of a normal distribution. To test for differences in mean $r^{2}$ between RG and WFG with RRSS methods, we excluded 54 "no flux" regressions (Pedersen et al 2010) that showed insignificant deviations from ambient $\mathrm{CH}_{4}$ concentration over the four sample points since relatively minor concentration differences stemming from experimental or analytical error often produced a low $r^{2}$ value unrelated to chamber or soil disturbance during setup or sampling. We used the non-parametric Mann-Whitney U test on rank-transformed $r^{2}$ absolute values from the remaining linear regressions since the distributions of $r^{2}$ absolute values do not adequately meet the normal distribution parameter required by $t$ tests.

Since we sampled from WFG chambers with the RRSS subsequent to our sampling from RG chambers (rather than a side-by-side comparison), it is necessary to investigate whether site conditions can explain differences in performance results. We consider water level and chamber $\mathrm{T}$ as potentially important site condition variables because each of them has potential to influence the pressure on interstitial soil gases and ebullition. To evaluate the relative importance of site conditions on $\mathrm{CH}_{4}$ data quality, we compared the mean and standard deviation of standing water level 
and $\mathrm{T}$ between static chamber methods. We compared chamber T recorded between methods using a Welch's $t$ test. Conventional statistical tests are not appropriate for water level, which contains excessive zeros (indicating water below the soil surface), so we converted the water level data to a binary factor indicating the presence/absence of ponded water. We tested whether the presence/absence of ponded water or chamber design had a more important impact of logtransformed $\mathrm{t}_{0} \mathrm{CH}_{4}$ concentration using a 2-way analysis of variance (ANOVA) and Tukey's honest significant difference test.

\section{Results}

Incubations from selected plots (sampled 21 October 2011 using RG; 26 September 2012 using WFG with RRSS) illustrate the differences in data reliability typically observed between the two sampling methods. RG static chambers often produced $C_{\text {initial }}$ values that were inflated one to three orders of magnitude above ambient $\mathrm{CH}_{4}$ concentration with non-linear $\mathrm{CH}_{4}$ accumulation over time (Fig. 2a). In contrast, WFG static chambers sampled using the RRSS regularly produced circum-ambient $C_{\text {initial }}$ values and linear slopes (Fig. 2b).

The use of RG static chambers produced a $C_{\text {initial }}$ greater than $3.6 \mathrm{ppm}$ (double ambient $\mathrm{CH}_{4}$ concentration) in 44 out of $67(66 \%)$ incubations and a $C_{\text {initial }}$ greater than 18 ppm (10 times ambient $\mathrm{CH}_{4}$ concentration) in 30 out of $67(45 \%)$ incubations compared to 6 out of $100(6 \%)$ and 0 out of $100(0 \%)$ when using WFG static chambers with RRSS (see Fig. 3a). Welch's $t$ test measured significantly higher mean $C_{\text {initial }}$ values $(p<0.0001)$ from $\mathrm{RG}$ compared to WFG static chambers with RRSS.

The use of RG static chambers produced $r^{2}$ greater than 0.9 for just 29 out of 64 (45\%) non-"no flux" linear regressions compared to 42 out of $49(86 \%)$ for those of the WFG static chamber with RRSS (see Fig. 3b). Mann-Whitney $U$ test found significantly lower mean $r^{2}$ values $(p<0.0001)$ from RG compared to WFG static chambers with RRSS.

To determine if environmental site conditions found in 2011 versus 2012 can explain the differences in performance results between the RG compared to WFG static chambers with RRSS, we compared water tables and soil temperatures. We found that chamber $\mathrm{T}$ was similar during RG and WFG static chamber sampling both years, with means ( \pm standard deviation) of $21.6( \pm 3.3)$ and $20.5( \pm 7.0)$ degrees $C$, respectively. A Welch's $t$ test found the difference in mean chamber $\mathrm{T}$ to not be significant $(p=0.18)$. Water level data suggest that conditions may have been wetter during RG static chamber sampling compared to WFG, with mean ( \pm standard deviation) standing water of $1.8 \mathrm{~cm}( \pm 2.8)$ and $0.8 \mathrm{~cm}( \pm 1.7)$ $\mathrm{cm}$, respectively. Ponded water was present for 32 of 67 (48 \%) RG chamber incubations compared to just 30 out of 100 (30\%) of WFG chamber incubations. However, a 2-way ANOVA and Tukey's honest significant difference test found that differences in $C_{\text {initial }}$ were related to sampling method $(p=0.0001)$ rather than the presence/absence of ponded water $(p=0.86)$.

We tested whether it was necessary to use our RRSS with the WFG by sampling half the WFG chambers without the RRSS in September 2012. In these 10 incubations, the RRSS system never produced $C_{\text {initial }}$ values greater than $3.6 \mathrm{ppm}$. When we did not use the RRSS, in contrast, all $C_{\text {initial }}$ values were greater than $3.6 \mathrm{ppm}$ with 5 out of 10 being greater than $18 \mathrm{ppm}$ (see Fig. 4). The Welch's $t$ test found that the RRSS produced significantly lower $(p<0.001)$ mean $C_{\text {initial }}$ values. When we used the RRSS, 9 out of 9 incubations that did not meet "no flux" criteria produced $r^{2}$ values greater than 0.9 compared to just 7 out of 10 when we did not use the RRSS for setup. While, the Mann-Whitney U test for a difference in mean $r^{2}$ values did not produce sufficient evidence to reject the null, the comparison shows that more samples will be lost when the RRSS system is not used.

\section{Discussion}

In studies of $\mathrm{CH}_{4}$ flux from wetlands, static chamber design and setup can have a huge influence on data reliability and therefore on the efficiency of data collection effort. The use of a RG-style static chamber (Weishampel and Kolka 2008) or any design that requires a close approach during chamber setup and sampling has the potential to cause soil compression resulting in high initial $\mathrm{CH}_{4}$ values. While disturbance may be imperceptible to a field observer, our data suggest it can artificially inflate $C_{\text {initial }}$ and/or reduce 
Fig. 2 Examples of gas data illustrating unsuccessful use of a rubber gasket static chamber design on 21 October 2011(a) and successful use of waterfilled-gutter static chamber design with remote rod sampling system on 26 September 2012 (b)
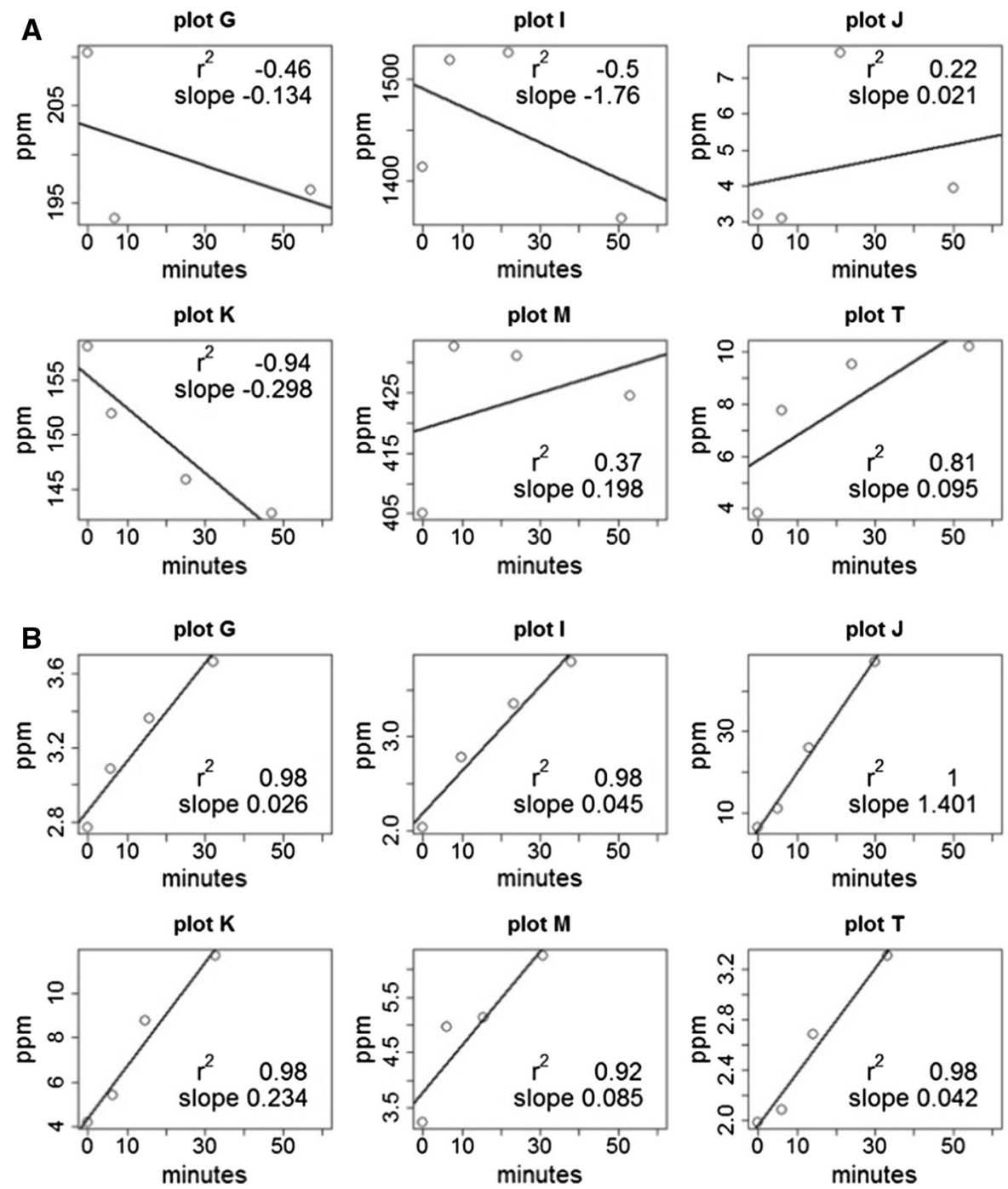

chamber setup suggest that elevated $C_{\text {initial }}$ can be caused by merely standing close to the chamber collars with either RG or WFG designs. Elevated $C_{\text {initial }}$ on its own may not necessarily lead to a decrease in $r^{2}$, but repeated approaches to the chamber during each of the four sampling time points, as we did before implementing the RRSS, significantly compromised regression linearity, thus leading to a rejection of a large number of measurements.

For decades, researchers have discussed optimal chamber design for achieving precision and accuracy in the estimation of gas flux from soils (Hutchinson and Mosier 1981; Anthony et al 1995; Conen and Smith 2000; Forbrich et al 2010). The data we present here demonstrate that disturbance of wetland soil 

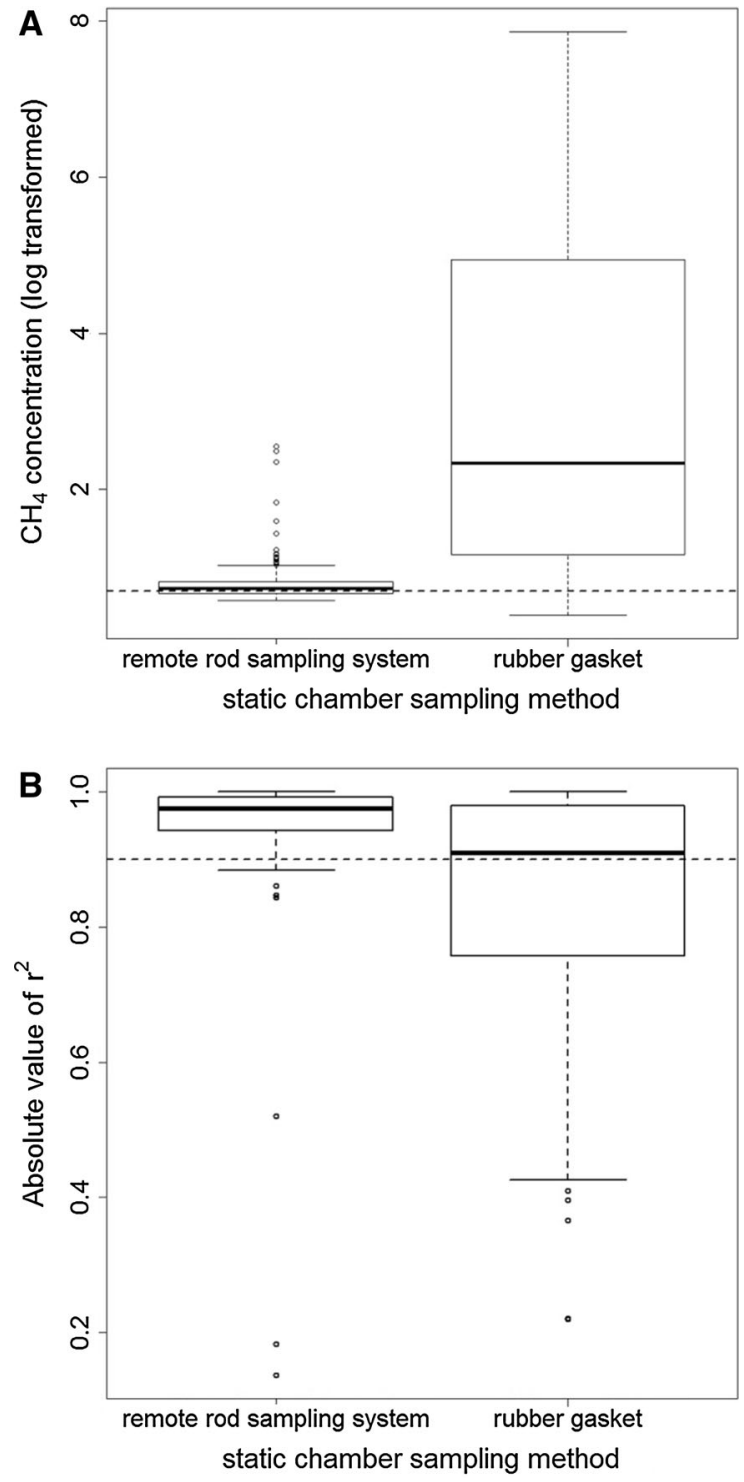

Fig. 3 Tukey boxplots of log-transformed initial $\mathrm{CH}_{4}$ concentrations in chamber headspace (a) and $r^{2}$ value of linear regression line used to estimate $\mathrm{CH}_{4}$ flux (b) from 67 incubations using the rubber gasket static chamber design taken from September 2011 through February 2012, and 110 incubations using the water-filled gutter with remote rod sampling system method taken from May 2012 through January 2013. Dashed lines mark ambient $\mathrm{CH}_{4}$ concentration (a) and $r^{2}$ threshold of 0.9 (b)

around static chambers can be effectively avoided by employing a WFG that allows for chambers to be set up and gas to be sampled using a RRSS to prevent soil disturbance. We suggest that disturbance is far more likely to impair data reliability than other potential

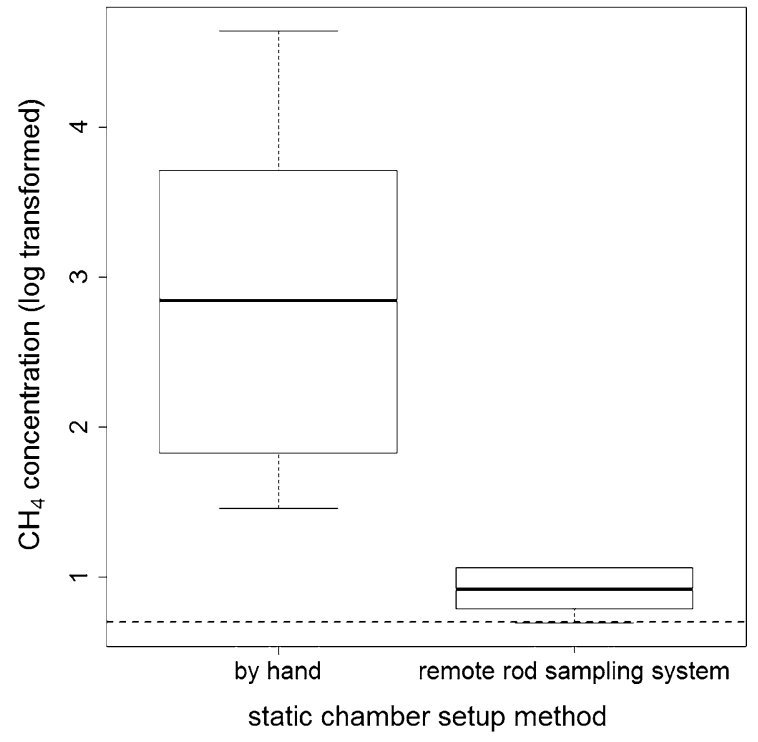

Fig. 4 Tukey boxplot of log-transformed initial $\mathrm{CH}_{4}$ concentration in chamber headspace from 10 incubations using remote rod sampling system to setup the chamber cap to minimize disturbance and from 1110 incubations for which the chamber cap setup by hand. Dashed line marks ambient $\mathrm{CH}_{4}$ concentration

sources of error endemic to the static chamber method, such as slight variations in internal chamber pressure due to extracted sample volume.

Chambers measured continuously using laser offaxis integrated cavity output spectroscopy (Mastepanov et al 2008), automated flux chambers (Scott et al 1999), and eddy covariance towers (Kormann et al 2001) are viable alternative methods for estimating methane flux from wetlands while avoiding soil disturbance, but they can increase costs by two or three orders of magnitude and the latter may not be appropriate for small footprint applications. The manual static chamber method remains popular because of its efficiency and cost-effectiveness, yet in a wetland setting a standard practice to avoid soil disturbance during sampling is to construct boardwalks, which can be costly, labor intensive, and logistically impractical. Our newly developed RRSS circumvents the need for boardwalks.

Acknowledgments We acknowledge the help of Jonathan Bills, who provided critical assistance with site instrumentation and field sampling. Hongjun Wang and Wes Willis assisted with configuration and maintenance of the gas chromatograph. Hongjun Wang, Ashley Helton, and Emily Bernhardt gave valuable advice and training in static chamber design and gas 
sampling and Todd Smith assisted with chamber construction. James Perry, Lee Daniels, David Bailey, and Leo Snead provided valuable insight into the site history and scope of previous investigations. We thank Ann and Jay Kinney for graciously allowing us to use of their driveway to access the site. Three anonymous reviewers provided helpful comments that improved this manuscript.

Funding This work was funded by the Peterson Family Foundation, Wetland Studies and Solutions Inc. and the Duke University Wetland Center Endowment.

\section{Compliance with Ethical Standards}

Conflict of Interest We declare no conflict of interest.

\section{References}

Anthony WH, Hutchinson GL, Livingston GP (1995) Chamber measurement of soil-atmosphere gas exchange: linear versus diffusion-based flux models. Soil Sci Soc Am J 59:1308. doi:10.2136/sssaj1995.03615995005900050015x

Bailey D, Perry J, Daniels W (2007) Vegetation dynamics in response to organic matter loading rates in a created freshwater wetland in southeastern Virginia. Wetlands 27:936-950

Bergschneider CR (2005) Determining an appropriate organic matter loading rate for a created coastal plain forested wetland. 91

Bridgham SD, Patrick Megonigal J, Keller JK et al (2006) The carbon balance of North American wetlands. Wetlands 26:889

Bruland G, Richardson C (2004) Hydrologic gradients and topsoil additions affect soil properties of Virginia created wetlands. Soil Sci Soc Am J 68:2069-2077

Christiansen JR, Korhonen JFJ, Juszczak R et al (2011) Assessing the effects of chamber placement, manual sampling and headspace mixing on $\mathrm{CH}_{4}$ fluxes in a laboratory experiment. Plant Soil 343:171-185. doi:10.1007/ s11104-010-0701-y

Conen F, Smith K (2000) An explanation of linear increases in gas concentration under closed chambers used to measure gas exchange between soil and the atmosphere. Eur J Soil Sci 51:111-117

Forbrich I, Kutzbach L, Hormann A, Wilmking M (2010) A comparison of linear and exponential regression for estimating diffusive $\mathrm{CH}_{4}$ fluxes by closed-chambers in peatlands. Soil Biol Biochem 42:507-515. doi:10.1016/j. soilbio.2009.12.004

Hutchinson G, Mosier A (1981) Improved soil cover method for field measurement of nitrous oxide fluxes. Soil Sci Soc Am J 45:311-316
Kormann R, Müller H, Werle P (2001) Eddy flux measurements of methane over the fen "Murnauer Moos", $1111^{\prime} \mathrm{E}, 47$ $39^{\prime} \mathrm{N}$, using a fast tunable diode laser spectrometer. Atmos Environ 35:2533-2544

Krauss KW, Whitbeck JL (2011) Soil greenhouse gas fluxes during wetland forest retreat along the Lower Savannah River, Georgia (USA). Wetlands 32:73-81. doi:10.1007/ s13157-011-0246-8

Livingston GP, Hutchinson GL (1995) Enclosure-based measurement of trace gas exchange: applications and sources of error. In: Matson A, Harriss RC (eds) Biogenic trace gases: measuring emissions from soil and water. Blackwell Science, Cambridge, pp 14-51

Mastepanov M, Sigsgaard C, Dlugokencky EJ et al (2008) Large tundra methane burst during onset of freezing. Nature 456:628-630. doi:10.1038/nature07464

Morse J, Ardon M, Bernhardt ES (2012) Greenhouse gas fluxes in southeastern US coastal plain wetlands under contrasting land uses. Ecol Appl 22:264-280

Myhre G, Shindell D, Bréon F-M et al (2013) Anthropogenic and natural radiative forcing. In: Stocker TF, Qin D, Plattner G-K et al (eds) Climate change 2013: the physical science basis. Cambridge University Press, Cambridge, pp 1-124

Nahlik AM, Mitsch WJ (2010) Methane emissions from created riverine wetlands. Wetlands 30:783-793. doi:10.1007/ s13157-010-0038-6

Parkin TB, Venterea RT (2010) USDA-ARS GRACEnet Project Protocols Chapter 3. Chamber-Based Trace Gas Flux Measurements 4. USDA-ARS GRACEnet Project Protocols 2010:1-39

Pedersen AR, Petersen SO, Schelde K (2010) A comprehensive approach to soil-atmosphere trace-gas flux estimation with static chambers. Eur J Soil Sci 61:888-902. doi:10.1111/j. 1365-2389.2010.01291.x

Scott A, Crichton I, Ball BC (1999) Long-term monitoring of soil gas fluxes with closed chambers using automated and manual systems. J Environ Qual 28:1637. doi:10.2134/ jeq1999.00472425002800050030x

Strack M, Kellner E, Waddington JM (2005) Dynamics of biogenic gas bubbles in peat and their effects on peatland biogeochemistry. Global Biogeochem Cycles 19:1-9. doi:10.1029/2004GB002330

Wang H, Lu J, Wang W et al (2006) Methane fluxes from the littoral zone of hypereutrophic Taihu Lake, China. J Geophys Res 111:1-8. doi:10.1029/2005JD006864

Weishampel P, Kolka R (2008) Measurement of methane fluxes from terrestrial landscapes using static, non-steady state enclosures. In: Hoover CM (ed) Field measurements for forest carbon monitoring. Springer, New York, pp 163-170

Winton RS, Richardson CJ (2015) The effects of organic matter amendments on greenhouse gas emissions from a mitigation wetland in Virginia's coastal plain. Wetlands. doi:10. 1007/s13157-015-0674-y 
Reproduced with permission of the copyright owner. Further reproduction prohibited without permission. 